\title{
Graphene nanomesh transistor with high on/off ratio and good saturation behavior
}

Salim Berrada, Viet Hung Nguyen, Damien Querlioz, Jérôme Saint-Martin, Alfonso Alarcón, Christophe Chassat, Arnaud Bournel, and Philippe Dollfus

Citation: Appl. Phys. Lett. 103, 183509 (2013); doi: 10.1063/1.4828496

View online: https://doi.org/10.1063/1.4828496

View Table of Contents: http://aip.scitation.org/toc/apl/103/18

Published by the American Institute of Physics

\section{Articles you may be interested in}

Disorder effects on electronic bandgap and transport in graphene-nanomesh-based structures Journal of Applied Physics 113, 013702 (2013); 10.1063/1.4772609

Direct growth of graphene nanomesh using a Au nano-network as a metal catalyst via chemical vapor deposition Applied Physics Letters 103, 023105 (2013); 10.1063/1.4813318

DNA-decorated graphene nanomesh for detection of chemical vapors Applied Physics Letters 103, 183110 (2013); 10.1063/1.4827811

Transfer patterning of large-area graphene nanomesh via holographic lithography and plasma etching Journal of Vacuum Science \& Technology B, Nanotechnology and Microelectronics: Materials, Processing, Measurement, and Phenomena 32, 06FF01 (2014); 10.1116/1.4895667

High-efficiency graphene nanomesh magnets realized by controlling mono-hydrogenation of pore edges Applied Physics Letters 104, 252410 (2014); 10.1063/1.4885390

SU-8 doped and encapsulated n-type graphene nanomesh with high air stability Applied Physics Letters 103, 232113 (2013); 10.1063/1.4841615

\section{LakeShore} CRYOTRONICS

\section{Sensors, Controllers, Monitors}

from the world leader in cryogenic thermometry 


\title{
Graphene nanomesh transistor with high on/off ratio and good saturation behavior
}

\author{
Salim Berrada, ${ }^{1, a)}$ Viet Hung Nguyen, ${ }^{1,2, b)}$ Damien Querlioz, ${ }^{1}$ Jérôme Saint-Martin, ${ }^{1}$ \\ Alfonso Alarcón, ${ }^{1}$ Christophe Chassat, ${ }^{1}$ Arnaud Bournel, ${ }^{1}$ and Philippe Dollfus ${ }^{1, a)}$ \\ ${ }^{1}$ Institute of Fundamental Electronics (IEF), Univ. Paris-Sud, CNRS, UMR 8622 Orsay, France \\ ${ }^{2}$ Institute of Physics, Center for Computational Physics, Vietnamese Academy of Science and Technology, \\ Hanoi, Vietnam
}

(Received 6 August 2013; accepted 14 October 2013; published online 31 October 2013)

\begin{abstract}
We investigate the device operation and performance of transistors based on a graphene nanomesh lattice. By means of numerical simulation, we show that this device architecture allows suppressing the chiral tunneling, which reduces drastically the off current and enhances the on/off ratio compared to the pristine graphene counterpart. Additionally, a good saturation of current can be reached in the thermionic regime of transport. Though reduced compared to the case of pristine transistors, the transconductance and the cutoff frequency are still high. Above all, the nanomesh transistors outperform their pristine graphene counterpart in terms of voltage gain and maximum oscillation frequency. (C) 2013 AIP Publishing LLC. [http://dx.doi.org/10.1063/1.4828496]
\end{abstract}

The exceptional intrinsic electronic properties of graphene as high carrier mobility ${ }^{1,2}$ and high critical current density $^{3}$ make this material an excellent candidate for highfrequency applications. ${ }^{4}$ Graphene transistors operating in the radio-frequency range have been demonstrated with graphene reported on sapphire substrate ${ }^{5}$ or with ChemicalVapor-Deposition (CVD)-grown graphene reported on diamond-like carbon substrate. ${ }^{6,7}$ Intrinsic cut-off frequencies above $300 \mathrm{GHz}$ and $400 \mathrm{GHz}$ have been reached with a $40 \mathrm{~nm}$ top-gate transistor on graphene epitaxially grown on $\mathrm{SiC}^{7}$ and with self-aligned transistors obtained by transferred gate stacks, ${ }^{8}$ respectively. A record maximum oscillation frequency of $70 \mathrm{GHz}$ has been recently reported for transistors with epitaxial graphene grown on the C-face of SiC. ${ }^{9}$ However, with a view to many analogue applications, the characteristics of graphene transistors are severely affected by a poor saturation of current leading to rather small voltage gain. Additionally, the lack of bandgap in graphene is known to make it difficult to turn off the current, which leads to $I_{O N} / I_{O F F}$ ratios lower than 10 , i.e., unacceptably small for digital applications. Different options have been proposed to overcome this limitation with alternative device concepts as a tunneling transistor based on vertical heterostructures, ${ }^{10}$ a gate-controlled Schottky barrier transistor, ${ }^{11}$ a hot-electron transistor ${ }^{12}$ or a transistor with physical gap designed on an impact-ionized Si MetalOxide-Semiconductor structure. ${ }^{13}$ To improve the off-state behavior in more conventional field-effect architecture, it is necessary to open a bandgap in graphene.

The most common approach consists in cutting 2D graphene sheets into 1D narrow nanoribbons (GNRs). By using $\mathrm{Ni}$ nanobars to grow GNRs, it has been possible to design GNR field-effect transistors (GNRFETs) with high on/off ratio at low temperature. ${ }^{14}$ However, for the bandgap to be

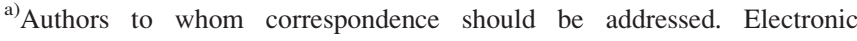
addresses: salim.berrada@u-psud.fr and philippe.dollfus@u-psud.fr

${ }^{b}$ Present address: L_Sim, SP2M, UMR-E CEA/UJF-Grenoble 1, INAC, Grenoble, France.
}

significant, the GNR width should be smaller than $3 \mathrm{~nm}$, which is difficult to achieve. Such small width gives rise to problems of reproducibility and edge disorder control. ${ }^{15,16}$ Additionally, it provides limited driving current. Opening bandgap in $2 \mathrm{D}$ graphene sheets is theoretically possible in the case of Bernal stacking of graphene on hexagonal boronnitride (BN) sheet. Bandgaps of $53 \mathrm{meV}$ and $100 \mathrm{meV}$ have been predicted ${ }^{17,18}$ and may even increase up to more than $200 \mathrm{meV}$ under external pressure. ${ }^{19,20}$ Additionally, when graphene is reported on h-BN, the interface is clean enough to make the mobility almost as high as in suspended graphene, ${ }^{2}$ which should allow reaching ballistic transport at room temperature. In the case of Bernal-stacking bilayer graphene, the vertical electric field that can be induced in a double-gate structure may generate a controllable bandgap of up to $250 \mathrm{meV}^{21,22}$ Bilayer graphene transistors have shown an improved saturation behavior. ${ }^{23}$ The possibility to open a bandgap in nitrogen-doped graphene has been also demonstrated recently. ${ }^{24}$

Alternatively, the opening of a bandgap in large sheets of graphene has been demonstrated by punching a high-density array of periodic nanoholes. ${ }^{25,26}$ Depending on the neck width, the meshing orientation and the shape of holes, bandgaps higher than $0.5 \mathrm{eV}$ could be achieved. ${ }^{27,28}$ Some authors have investigated quite systematically the bandgap opening in such perfect graphene nanomesh (GNM) lattices ${ }^{29,30}$ and suggested some scaling rules, showing a strong sensitivity to the hole shape and lattice orientation. However, the unavoidable effect of disorder has been shown to reduce strongly the sensitivity to the hole shape and to simplify the scaling rule in the form $E_{G}=\alpha / W_{n w},{ }^{28}$ where $\alpha$ is a constant and $W_{n w}$ is the neck width, in agreement with experimental data. ${ }^{26}$ It opens new routes of band structure engineering for graphene applications, including tunnel diodes, ${ }^{31}$ waveguides, ${ }^{32}$ and magnetic structures. ${ }^{33}$ In principle, this method offers the additional advantage of making possible to generate positiondependent bandgaps, i.e., to introduce a bandgap only in regions of the graphene sheet where it is useful for device optimization without degrading strongly the transport 
properties. ${ }^{28,31}$ Here, we exploit this possibility by introducing a GNM section in the gated part of short-channel graphene FETs. It allows us to suppress efficiently the chiral tunneling through the gate-induced potential barrier and to enhance drastically the $\mathrm{I}_{\mathrm{ON}} / \mathrm{I}_{\mathrm{OFF}}$ ratio compared to pristine graphene FET. Improved saturation behavior and good high frequency parameters are also obtained.

Our model is based on a nearest-neighbor tight-binding (TB) Hamiltonian to describe the graphene honeycomb lattice, ${ }^{34}$ using $t=2.7 \mathrm{eV}$ as hopping energy. ${ }^{35}$ For large graphene sheets with uniform or nanomesh lattice, where the lateral width of the device can be considered to be much larger than the channel length, the width direction $y$ can be considered through Bloch periodic boundary conditions. ${ }^{36}$ The lattice is then split into unit cells and by Fourier transform along the Oy direction, the 2D TB Hamiltonian is rewritten in the form of the sum of decoupled 1D Hamiltonians $\tilde{H}_{1 D}\left(k_{y}\right)$ for each discretized value of wave vector $k_{y}{ }^{28}$ The Green's functions of these Hamiltonians are solved in the ballistic limit self-consistently with the 3D Poisson's equation, which gives us the steady-state momentum- and energydependent transmission probabilities $T\left(E, k_{y}\right) .{ }^{37}$ The current is finally computed from the Landauer equation as

$$
I=\frac{e}{\pi h} \int_{-\infty}^{\infty} d E \int_{B Z} d k_{y} T\left(E, k_{y}\right)\left[f_{S}(E)-f_{D}(E)\right],
$$

where $f_{S(D)}(E)$ is the source(drain) Fermi distribution function and the integral over $k_{y}$ is performed in the first Brillouin zone.

The simulated devices are schematized in Fig. 1. The monolayer graphene sheet is assumed to lie on a h-BN substrate, which is known to lead to high mobility and mean free path and makes the ballistic approximation justified. The source and drain access regions are assumed to be electrostatically doped by a back gate up to an N-type density of $10^{13} \mathrm{~cm}^{-2}$. The $30 \mathrm{~nm}$-long gated region is made of GNM lattice. For comparison, a full pristine graphene transistor of same gate length has been simulated as a reference. We have considered three GNM perfect square lattices of hexagonal nano-holes of different size and neck widths. Their parameters are summarized in Table I. The corresponding bandgaps are $268 \mathrm{meV}, 508 \mathrm{meV}$, and $553 \mathrm{meV}$, with effective mass in the first subband of $0.074 m_{0}, 0.064 m_{0}$, and $0.113 m_{0}$, respectively.

In pristine graphene transistor (GFET), one can identify three different transport regimes depending on the energy range of the current window $\left[E_{F D}, E_{F S}\right] .{ }^{38}$ (i) For energies

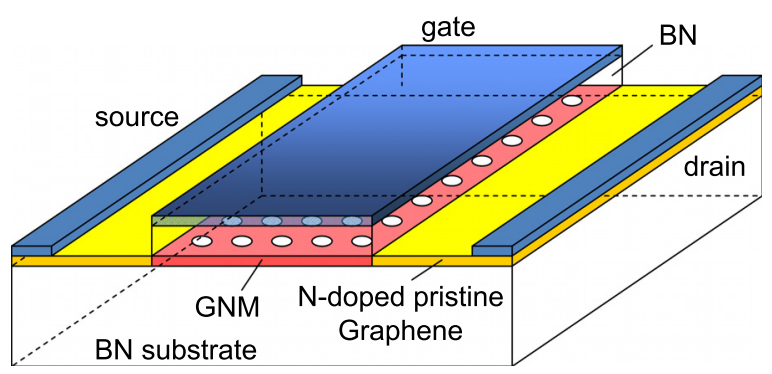

FIG. 1. Schematic view of the GNM transistor considered in this work.
TABLE I. Description of GNM lattices with hexagonal holes used in this work for transistor simulation. $W_{x}$ and $W_{y}$ are the neck widths (distances between holes) and $E_{G}$ is the resulting bandgap.

\begin{tabular}{lccc}
\hline \hline $\begin{array}{l}\text { Number of missing } \\
\text { atoms in the holes }\end{array}$ & $\begin{array}{c}W_{x} \\
(\mathrm{~nm})\end{array}$ & $\begin{array}{c}W_{y} \\
(\mathrm{~nm})\end{array}$ & $\begin{array}{c}E_{G} \\
(\mathrm{meV})\end{array}$ \\
\hline 6 & 1 & 1.1 & 508 \\
24 & 1.1 & 1.2 & 553 \\
54 & 2.3 & 2.2 & 268 \\
\hline \hline
\end{tabular}

above the potential barrier induced by the gate voltage $V_{G S}$, the thermionic transmission is the classical regime of transport in conventional semiconductor FETs when the different sources of tunneling leakage have negligible contributions. (ii) At low energy, as in small bandgap semiconductors or under high drain voltage $V_{D S}$, the band-to-band tunneling (BTBT) takes place between the valence band in the source and the conduction band in the drain. (iii) At intermediate energy, the chiral tunneling of particles through the barrier is specific of graphene and gives rise to Klein (or chiral) tunneling. ${ }^{39,40}$ The chiral tunneling may be strong and even dominant at negative $V_{G S}$, as a consequence of the good matching of electron states in the source and hole states in the barrier. In Fig. 2, we plot in dashed lines the different contributions to the current in the pristine GFET as a function of $V_{G S}$, for $V_{D S}=0.3 \mathrm{~V}$. The contribution of BTBT is negligible, much lower than the contributions of either chiral tunneling ( $\mathrm{p}$-branch) or thermionic transmission (n-branch). Actually, the contribution of BTBT is significant only at higher $V_{D S}$. Hence, the Dirac point in the $I_{D}-V_{G S}$ characteristics corresponds to the point of equilibrium between chiral tunneling and thermionic transmission (point A in Fig. 2). The chiral tunneling component is clearly responsible for an off-current of GFETs that is known to be unacceptably high for digital applications. ${ }^{38}$ To improve this behavior, the first requirement is to suppress significantly this contribution of chiral tunneling. It is the main motivation behind the proposal of GNM transistor, or GNM-FET. A GNM section is introduced in the gated part of the transistor to make the potential barrier much less transparent to chiral particles.

This effect is clearly shown in Fig. 3 where we plot the local density of states (LDOS, left panel)) and the energy spectrum of current (right panel) at the Dirac point $\left(V_{G S}=0.1 \mathrm{~V}\right.$, $V_{D S}=0.2 \mathrm{~V}$ ) for the GNM-FET with $E_{G}=508 \mathrm{meV}$. The potential profile at the neutrality point is superimposed to the LDOS. The oscillations of potential in the channel are due to the influence of nanoholes of the GNM lattice. The bandgap in the gated region overlaps fully the energy window of chiral tunneling and makes impossible the chiral transmission of particles through the barrier. It is confirmed by the current spectrum: the only contributions to the current come from the thermionic transmission and the BTBT. It is in contrast with the results obtained in pristine transistors, as shown for instance in Fig. 2 of Ref. 38. The effect of bandgap on the chiral tunnelling close to the Dirac point can be summarized as follows: for $V_{G S}=0.1 \mathrm{~V}$ and $V_{D S}=0.2 \mathrm{~V}$, this current component falls from $265 \mu \mathrm{A} / \mu \mathrm{m}$ for $E_{G}=0$, to $10.6 \mu \mathrm{A} / \mu \mathrm{m}$ for $E_{G}=268 \mathrm{meV}, 4.6 \times 10^{-3} \mu \mathrm{A} / \mu \mathrm{m}$ for $E_{G}=508 \mathrm{meV}$ and $2.3 \times 10^{-3} \mu \mathrm{A} / \mu \mathrm{m}$ for $E_{G}=553 \mathrm{meV}$. 




FIG. 2. The different contributions to the current in pristine (dashed lines) and GNM (solid lines) transistors as a function of the gate voltage, for the drain voltage $V_{D S}=0.2 \mathrm{~V}$. In the latter case, the GNM lattice bandgap is equal to $508 \mathrm{meV}$.

The bandgap reduces also strongly the thermionic current, especially close to the Dirac point of the $I_{D}-V_{G S}$ characteristics, as shown in Fig. 2 where we plot in solid lines the different contributions to the current (in the case $E_{G}=508 \mathrm{meV}$ ), in comparison with that of the pristine GFET (dashed lines). The immediate consequence of the bandgap-induced suppression of both chiral tunneling and thermionic transmission is shown in the $I_{D}-V_{G S}$ characteristics of all simulated devices plotted in Fig. 4 for $V_{D S}=0.2 \mathrm{~V}$. When increasing $E_{G}$, the overall current is reduced but the off-current at the Dirac point is drastically suppressed. Actually, by defining arbitrarily the oncurrent as the current obtained at $V_{G S}=1.5 \mathrm{~V}$, the on/off ratio increases from 7 for pristine GFET to more than 7400 for GNM-FETs with $E_{G}>500 \mathrm{meV}$. Another important consequence of the suppression of chiral tunneling in the GNM devices is that the Dirac point in the transfer characteristics is now governed by the balance between thermionic and BTBT contributions, as shown in Fig. 2 (point B). It makes a strong difference of electrical behavior between pristine and GNM FETs, as shown in the transfer characteristics of Fig. 5.

In the p-branch of pristine FET, where the current is dominated by the chiral tunneling, it has been shown and explained that a pseudo-saturation of current, and even a negative differential conductance, may be observed under some
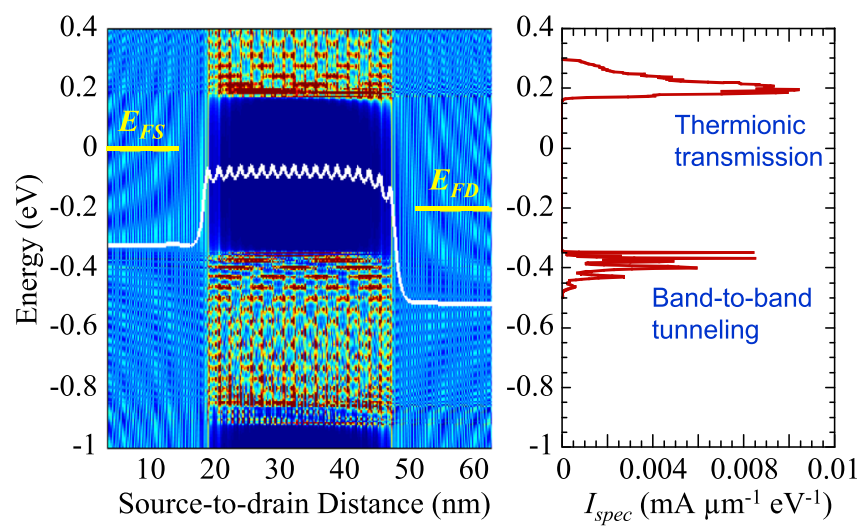

FIG. 3. (Left panel) Local Density of States in the GNM device with $E_{G}=508 \mathrm{meV}$ for $V_{G S}=0.1 \mathrm{~V}$ and $V_{D S}=0.2 \mathrm{~V}$ corresponding to a Dirac point of the transfer characteristics. The source and drain Fermi levels $E_{F S}$ and $E_{F D}$ are indicated and the potential profile at the neutrality point is superimposed. (right panel) Corresponding energy spectrum of current.

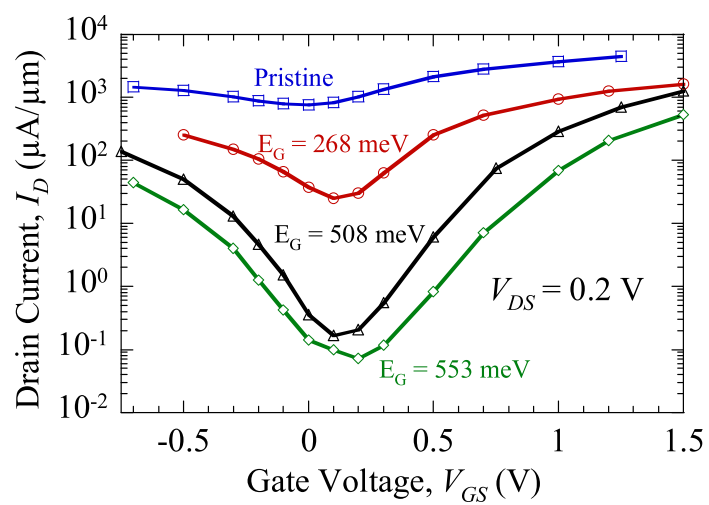

FIG. 4. Transfer characteristics of all simulated devices for $V_{D S}=0.2 \mathrm{~V}$.

conditions in a limited range of $V_{D S} \cdot{ }^{38,41}$ Similar effects are visible also in the GNM FET for very negative gate voltage $\left(V_{G S}<0.4 \mathrm{~V}\right)$. However, the most striking feature is that for moderate gate voltages of the p-branch the current is strongly influenced by the BTBT and thus increases when increasing $V_{D S}$. It is also the reason of the strong shift of the Dirac point towards positive values of $V_{G S}$ at increasing $V_{D S}$. The situation is very different in the n-branch. While the current continuously increases when increasing $V_{D S}$ in pristine FET due to a strong effect of drain-induced barrier lowering, ${ }_{G S}^{38}$ it becomes weakly $V_{D S}$-dependent on a large range of $V_{G S}$ in GNM-FET. A true saturation of current is thus observed in the latter, as shown more explicitly in the $I_{D^{-}} V_{D S}$ characteristics of Fig. 6. It is a remarkable achievement for a sub-50 nm graphene FET. Such a good saturation behavior has been observed previously in bilayer GFET with $4 \mu \mathrm{m}$ gate length thanks to the vertical field-induced bandgap. ${ }^{23}$

For radio frequency applications important figures of merit are the intrinsic transconductance $g_{m}$, the cutoff frequency $f_{T}$, the maximum oscillation frequency $f_{\max }$, and the voltage gain defined as $A_{v}=g_{m} / g_{d}$, where $g_{d}$ is the output conductance. $^{42,43}$ Conventional GFETs on monolayer graphene are usually considered as excellent candidates to provide high cutoff frequencies but their performance in terms of voltage gain is dramatically affected by a high output conductance, leading usually to $A_{v}$ smaller than 1 . Given the results observed above for GNM FETs, it is important to assess their performance regarding these parameters.

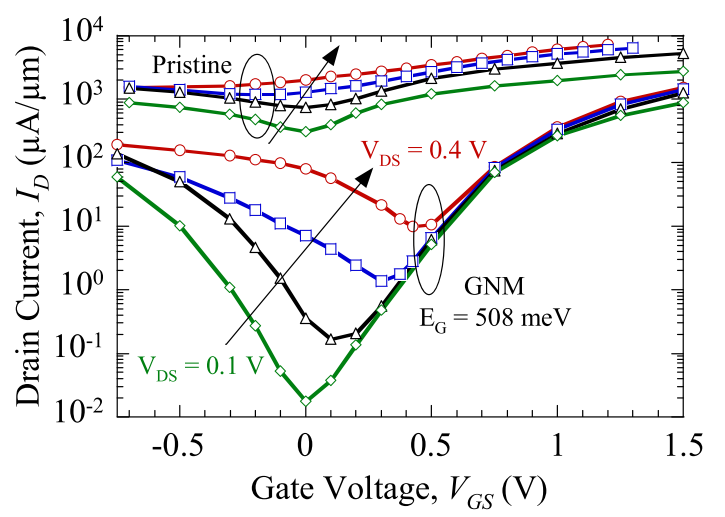

FIG. 5. Characteristics of pristine GFET and GNM-FET (with $E_{G}=508 \mathrm{meV}$ ) for different values of $V_{D S}$ ranging from $0.1 \mathrm{~V}$ to $0.4 \mathrm{~V}$, by steps of $0.1 \mathrm{~V}$. 


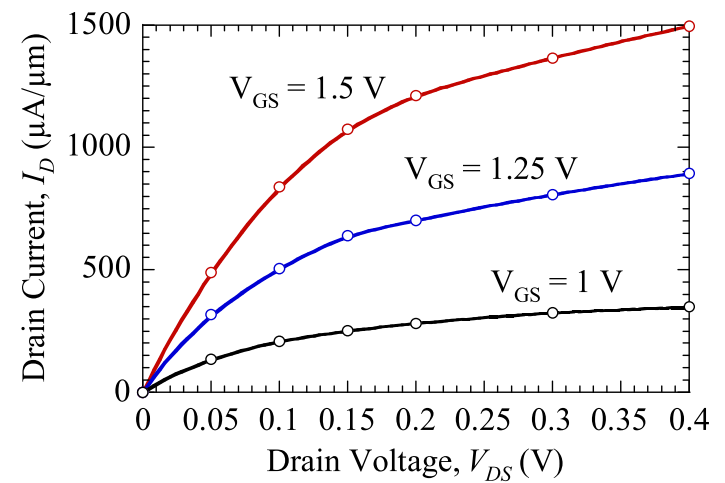

FIG. 6. $I_{D}-V_{D S}$ characteristics in the n-branch of GNM-FET with $E_{G}=508 \mathrm{meV}$.

In Figs. 7(a) and 7(b), we plot for both devices in the n-branch range of operation the transconductance and the cutoff frequency, respectively, for two values of $V_{D S}$. Here, $f_{T}$ is evaluated using the quasi-static approximation $f_{T}=g_{m} / 2 \pi C_{G}$, where $C_{G}$ is the total gate capacitance calculated as the derivative of the charge in the device with respect to $V_{G S}$. Though the maximum $g_{m}$ and $f_{T}$ are higher in pristine GFET than in GNM FET, it is remarkable that the loss of performance is very reasonable in the latter device. For instance, in GNM FET at $V_{D S}=0.2 \mathrm{~V}$, the maximum values of $g_{m}$ and $f_{T}$ reach $2305 \mu \mathrm{S} / \mu \mathrm{m}$ and $1.23 \mathrm{THz}$, respectively, i.e., $62 \%$ and $79 \%$, respectively, of the corresponding values for the pristine GFET. These fractions are still $56 \%$ and $63 \%$ at $V_{D S}=0.3 \mathrm{~V}$. However, thanks to the good saturation behavior discussed previously, the GNM FET outperforms its pristine counterpart in terms of output conductance and voltage gain. At $V_{D S}=0.3 \mathrm{~V}$ and $V_{G S}=1 \mathrm{~V}, A_{v}$ is limited to 0.32 in pristine
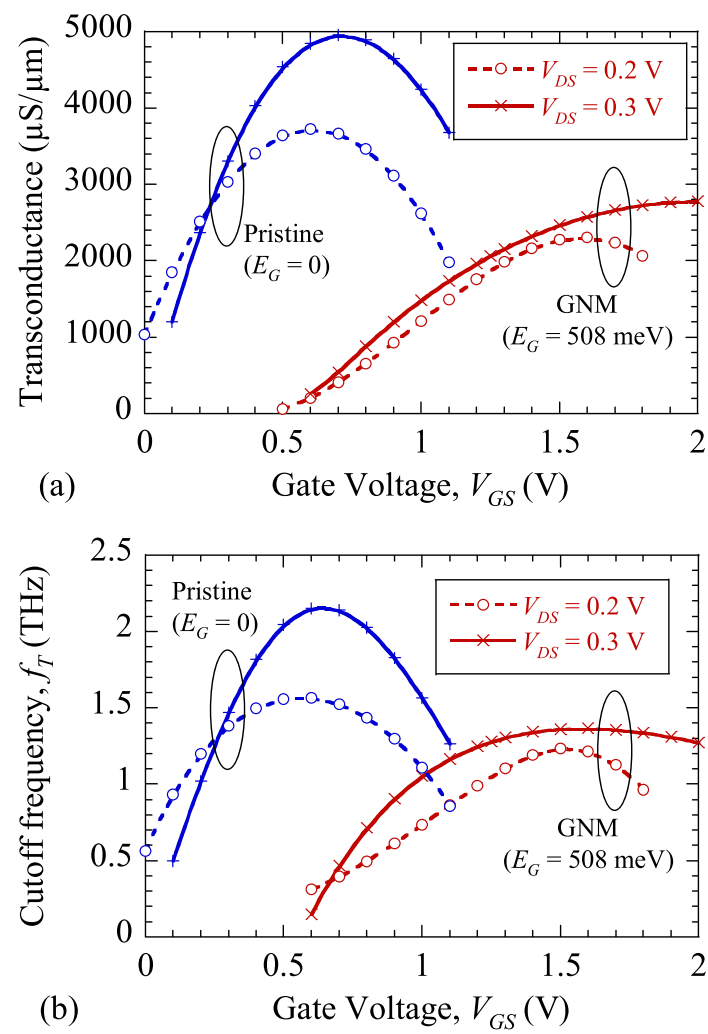

FIG. 7. (a) Transconductance $g_{m}$ and (b) cutoff frequency $f_{T}$ in pristine GFET and GNM-FET with $E_{G}=508 \mathrm{meV}$.
GFET and reaches 4.5 for GNM FET, which is a good performance for such small device.

Finally, we can evaluate the maximum oscillation frequency $f_{\max }$ from the expression $f_{\max } \approx f_{T} / \sqrt{\alpha+\beta f_{T}}$, where $\alpha=4 g_{D}\left(R_{S}+R_{G}\right), \beta=8 \pi C_{G D} R_{G}, C_{G D}$ is the gate-todrain capacitance, and $R_{S}$ and $R_{G}$ are the source and gate resistances, respectively. The simulation approach used here cannot provide information on $R_{S}$ and $R_{G}$. However, we may assume reasonable values, considering that the metal/graphene contact resistance leads typically to $R_{S} \approx 100 \Omega \mu \mathrm{m}$ (Ref. 44) and that $R_{G}$ of about $20 \Omega$ are reachable for graphene transistors of $20 \mu \mathrm{m}$ gate width. ${ }^{42}$ For the GNM device, we obtained $g_{D}=336 \mu \mathrm{S} / \mu \mathrm{m}$ with $C_{G D}=0.152 \mathrm{fF} / \mu \mathrm{m}$ at $V_{G S}=1 \mathrm{~V}$ and $V_{D S}=0.3 \mathrm{~V}$, the bias point for which the transition frequency reaches $f_{T}=1.365 \mathrm{THz}$. These parameters lead to the quantities $\alpha=0.672$ and $\beta=0.382 \mathrm{THz}^{-1}$, i.e., to $\beta f_{T}=0.521$ and the correction factor $\alpha+\beta f_{T}=1.193$. In the pristine GFET at the same bias point, the high output conductance $g_{D}=13360 \mu \mathrm{S} / \mu \mathrm{m}$ is the limiting factor of $f_{\max }$, with a parameter $\alpha$ reaching the value of 26.7 that dominates the correction factor. As a consequence, while $f_{\max }$ is still as high as $1.25 \mathrm{THz}$ in the GNM-FET, it falls to $0.41 \mathrm{THz}$ in the pristine GFET. The overall performance of the GNM-FET is finally much more attractive than that of its pristine counterpart, from both viewpoints of digital and analog applications.

In conclusion, thanks to its good saturation behavior and high on/off ratio, the GNM-FET proposed in this work offers strong advantages over its pristine graphene counterpart. Indeed, the GNM-induced bandgap allows limiting strongly the contributions of chiral tunneling and thermionic transmission at the Dirac point and controlling better the potential and the thermionic current in the n-branch of the $I_{D}-V_{G S}$ characteristics. Though the transconductance and transition frequency are reasonably smaller in the GNM-FET due to reduced transport properties, the latter device outperforms the pristine GFET in terms of output conductance, voltage gain, and maximum oscillation frequency.

One remaining issue that should be considered when designing GNM devices is related to the atomic edge disorder of holes. In principle, this disorder can affect detrimentally the output current. However, in a recent work, ${ }^{28}$ we demonstrated, by considering similar gate-induced potential barrier structures (but without the self-consistence with Poisson's equation), that the use of suitable GNM sections of finite length in the gated region makes it possible to avoid this effect to a large extent while good device performance is still obtained. Additionally, recent developments suggest that we may expect the disorder to be properly controlled soon. ${ }^{45}$

This work was partially supported by the French ANR through projects NANOSIM_GRAPHENE (ANR-09-NANO016) and MIGRAQUEL (ANR-10-BLAN-030403). One of the authors (VHN) acknowledges the Vietnam National Foundation for Science and Technology (NAFOSTED) for financial support under Project No. 103.02-2012.42.

${ }^{1}$ K. I. Bolotin, K. J. Sikes, J. Jiang, M. Klima, G. Fudenberg, J. Hone, P. Kim, and H. L. Stormer, Solid State Commun. 146, 351 (2008).

${ }^{2}$ P. J. Zomer, S. P. Dash, N. Tombros, and B. J. van Wees, Appl. Phys. Lett. 99, 232104 (2011). 
${ }^{3}$ K. S. Novoselov, A. K. Geim, S. V. Morozov, D. Jiang, Y. Zhang, S. V. Dubonos, I. V. Grigorieva, and A. A. Firsov, Science 306, 666 (2004).

${ }^{4}$ F. Schwierz, Nat. Nanotechnol. 5, 487 (2010).

${ }^{5}$ E. Pallecchi, C. Benz, A. C. Betz, H. V. Löhneysen, B. Plaçais, and R. Danneau, Appl. Phys. Lett. 99, 113502 (2011).

${ }^{6}$ Y. Wu, Y.-M. Lin, A. A. Bol, K. A. Jenkins, F. Xia, D. B. Farmer, Y. Zhu, and Ph. Avouris, Nature 472, 74 (2011).

${ }^{7}$ Y. Wu, K. A. Jenkins, A. Valdes-Garcia, D. B. Farmer, Y. Zhu, A. A. Bol, C. Dimitrakopoulos, W. Zhu, F. Xia, Ph. Avouris, and Y.-M. Lin, Nano Lett. 12, 3062 (2012).

${ }^{8}$ R. Cheng, J. Bai, L. Liao, H. Zhou, Y. Chen, L. Liu, Y. C. Lin, S. Jiang, Y. Huang, and X. Duan, Proc. Nat. Acad. Sci. USA 109, 11588 (2012).

${ }^{9}$ Z. Guo, R. Dong, P. S. Chakraborty, N. Lourenco, J. Palmer, Y. Hu, M. Ruan, J. Hankinson, J. Kunc, J. D. Cressler, C. Berger, and W. A. de Heer, Nano Lett. 13, 942 (2013).

${ }^{10}$ L. Britnell, R. V. Gorbachev, R. Jalil, B. D. Belle, F. Schedin, A. Mishchenko, T. Georgiou, M. I. Katsnelson, L. Eaves, S. V. Morozov, N. M. R. Peres, J. Leist, A. K. Geim, K. S. Novoselov, and L. A. Ponomarenko, Science 335, 947-950 (2012).

${ }^{11}$ H. Yang, J. Heo, S. Park, Hyun J. Song, D. H. Seo, K.-E. Byun, P. Kim, I. Yoo, H.-J. Chung, and K. Kim, Science 336, 1140 (2012).

${ }^{12}$ S. Vaziri, G. Lupina, C. Henkel, A. D. Smith, M. Ostling, J. Dabrowski, G. Lippert, W. Mehr, and M. C. Lemme, Nano Lett. 13, 1435-1439 (2013).

${ }^{13}$ J. H. Mun and B. J. Cho, Appl. Phys. Lett. 101, 143102 (2012).

${ }^{14}$ T. Kato and R. Hatakeyama, Nat. Nanotechnol. 7, 651 (2012).

${ }^{15}$ D. Querlioz, Y. Apertet, A. Valentin, K. Huet, A. Bournel, S. GaldinRetailleau, and P. Dollfus, Appl. Phys. Lett. 92, 042108 (2008).

${ }^{16}$ M. Y. Han, J. C. Brant, and P. Kim, Phys. Rev. Lett. 104, 056801 (2010).

${ }^{17}$ G. Giovannetti, P. A. Khomyakov, G. Brocks, P. J. Kelly, and J. van den Brink, Phys. Rev. B 76, 073103 (2007).

${ }^{18}$ N. Kharche and S. K. Nayak, Nano Lett. 11, 5274-5278 (2011).

${ }^{19}$ Y. Xu, Z. Guo, H. Chen, Y. Yuan, J. Lou, X. Lin, H. Gao, H. Chen, and B. Yu, Appl. Phys. Lett. 99, 133109 (2011).

${ }^{20}$ Y. Fan, M. Zhao, Z. Wang, X. Zhang, and H. Zhang, Appl. Phys. Lett. 98, 083103 (2011).

${ }^{21}$ F. Xia, D. B. Farmer, Y. M. Lin, and P. Avouris, 2010 Nano Lett. 10, 715 (2010).

${ }^{22}$ Y. Zhang, T. T. Tang, C. Girit, Z. Hao, M. C. Martin, A. Zettl, M. F. Crommie, Y. Ron Shen, and F. Wang, Nature 459, 820 (2009).

${ }^{23}$ B. N. Szafranek, G. Fiori, D. Schall, D. Neumaier, and H. Kurz, Nano Lett. 12, 1324 (2012).
${ }^{24}$ A. Lherbier, A. R. Botello-Méndez, and J.-C. Charlier, Nano Lett. 13, 1446-1450 (2013).

${ }^{25}$ J. Bai, X. Zhong, S. Jiang, Y. Huang, and X. Duan, Nat. Nanotechnol. 5, 190 (2010).

${ }^{26}$ X. Liang, Y. S. Jung, S. Wu, A. Ismach, D. L. Olynick, S. Cabrini, and J. Bokor, Nano Lett. 10, 2454 (2010).

${ }^{27}$ W. Oswald and Z. Wu, Phys. Rev. B 85, 115431 (2012).

${ }^{28}$ V. Hung Nguyen, M. Chung Nguyen, H. Viet Nguyen, and P. Dollfus, J. Appl. Phys. 113, 013702 (2013).

${ }^{29}$ T. G. Pedersen, C. Flindt, J. Pedersen, N. A. Mortensen, A.-P. Jauho, and K. Pedersen, Phys. Rev. Lett. 100, 136804 (2008).

${ }^{30}$ M. Dvorak, W. Oswald, and Z. Wu, Sci. Rep. 3, 2289 (2013).

${ }^{31}$ V. Hung Nguyen, F. Mazzamuto, J. Saint-Martin, A. Bournel, and P. Dollfus, Nanotechnology 23, 065201 (2012).

${ }^{32}$ J. P. Pedersen, T. Gunst, T. Markussen, and T. G. Pedersen, Phys. Rev. B 86, 245410 (2012).

${ }^{33}$ H. X. Yang, M. Chshiev, D. W. Boukhvalov, X. Waintal, and S. Roche, Phys. Rev. B 84, 214404 (2011).

${ }^{34}$ V. Hung Nguyen, V. Nam Do, A. Bournel, V. Lien Nguyen, and P. Dollfus, J. Appl. Phys. 106, 053710 (2009).

${ }^{35}$ S. Reich, J. Maultzsch, and C. Thomsen, Phys. Rev. B 66, 035412 (2002).

${ }^{36}$ G. Fiori and G. Iannaccone, IEEE Electron Device Lett. 30, 261 (2009).

${ }^{37}$ V. Hung Nguyen, J. Saint-Martin, D. Querlioz, F. Mazzamuto, A. Bournel, Y.-M. Niquet, and P. Dollfus, J. Comput. Electron 12, 85-93 (2013).

${ }^{38}$ A. Alarcón, V. Hung Nguyen, S. Berrada, D. Querlioz, J. Saint-Martin, A. Bournel, and P. Dollfus, IEEE Trans. Electron Devices 60, 985 (2013).

${ }^{39}$ M. I. Katsnelson, K. S. Novoselov, and A. K. Geim, Nat. Phys. 2, 620 (2006).

${ }^{40}$ V. Nam Do, V. Hung Nguyen, P. Dollfus, and A. Bournel, J. Appl. Phys. 104, 063708 (2008).

${ }^{41}$ R. Grassi, T. Low, A. Gnudi, and G. Baccarani, IEEE Trans. Electron. Devices 60, 140-146 (2013).

${ }^{42}$ B. Farmer, A. Valdes-Garcia, C. Dimitrakopoulos, and P. Avouris, Appl. Phys. Lett. 101, 143503 (2012).

${ }^{43}$ G. Fiori and G. Iannaccone, "Graphene RF design: what really matters," in Book of Abstracts GRAPHENE 2013, Bilbao, Spain, April 23-26, 2013.

${ }^{44}$ F. Xia, V. Perebeinos, Y.-M. Lin, Y. Wu, and Ph. Avouris, Nat. Nanotechnol. 6, 179 (2011).

${ }^{45}$ M. Wang, L. Fu, L. Gan, C. Zhang, M. Rummeli, A. Bachmatiuk, K. Huang, Y. Fang, and Z. Liu, Sci. Rep. 3, 1238 (2013). 